\title{
Sintering Induced Phase Transformation of Nanoparticles: A Molecular Dynamics Study
}

\author{
Qian Mao', Yihua Ren², Kai H. Luo ${ }^{1,3 *}$, Shuiqing Li $^{2}$ \\ ${ }^{1}$ Center for Combustion Energy, Key Laboratory for Thermal Science and Power Engineering of \\ Ministry of Education, Department of Thermal Engineering, Tsinghua University, Beijing 100084, \\ China
}

${ }^{2}$ Key Laboratory for Thermal Science and Power Engineering of Ministry of Education, Department of Thermal Engineering, Tsinghua University, Beijing 100084, China

${ }^{3}$ Department of Mechanical Engineering, University College London, Torrington Place, London WC1E 7JE, UK

\section{*Corresponding author:}

Telephone number: +44 (0)20 76793916

Fax number: $+44(0) 2073880180$

Email:K.Luo@ucl.ac.uk

Address: Department of Mechanical Engineering, University College London, Torrington Place,

London 


\begin{abstract}
Sintering induced phase transformation of $\mathrm{TiO}_{2}$ nanoparticles is investigated systematically via molecular dynamics simulation. By defining a coordination number and bond angle distribution criteria, local phase information is identified for each individual $\mathrm{Ti}$ atom originated from amorphous or crystal structure as well as three $\mathrm{TiO}_{2}$ polymorphs, anatase, brookite, and rutile. Size-dependent structures of nanoparticles lead to different dynamics of the sintering induced phase transformation. Grain boundaries that form between nanoparticles during sintering trigger the nucleation and growth of new phases. During the sintering of two equal-sized core-shell anatase nanoparticles, crystal core regions melt with the temperature rising and the surface energy decreasing in the microcanonical (NVE) ensemble. The new phase that develops from the grain boundary spreads into the destroyed core regions in stages, forming a new larger spherical nanoparticle with an ordered atomic arrangement. During the sintering of two unequal-sized nanoparticles (amorphous and core-shell anatase), atoms from the amorphous nanoparticle first nucleate to form crystal anatase in the contact region and a grain boundary is then developed between the original core region and the newly formed anatase crystal. After that, phase transformation follows much the same route as the equal-sized case from anatase to brookite.
\end{abstract}




\section{Introduction}

Phase stability of particles is a complex issue, which depends on a series of state variables such as external temperature, pressure, particle size, surface tension, etc. ${ }^{1}$ Based on the principles of thermodynamics, changes in the variables will have an influence on the Gibbs free energy, thus leading to transformation of phase as well as morphology of particles. ${ }^{2,3}$ However, when the particle size reaches the nanoscale, high surface curvature, core-shell structure, and the depression of the melting point of a nanoparticle significantly influence its phase stability. ${ }^{1,4-10}$ Experiments, first-principle calculations, and molecular dynamics (MD) simulations have revealed that anatase is thermodynamically most stable at the nanoscale among three typical $\mathrm{TiO}_{2}$ polymorphs, while rutile is stable in bulk and brookite is a metastable state in between. ${ }^{11-17}$ Experimental results reveal that morphologies, sizes, surface states, surface environment such as surface coating, the $\mathrm{pH}$ value of the solution all contribute to phase transformation of nanoparticles. ${ }^{1,17-21}$ In particular, the transition diameter for spherical $\mathrm{TiO}_{2}$ nanoparticles from anatase to rutile is about $2.6 \mathrm{~nm}$ in vacuum, as indicated by DFT calculation ${ }^{22}$ and MD simulations ${ }^{14,15}$.

Sintering, a common process during synthesis of nanoparticles, is usually accompanied by phase transformation. As-synthesized pure anatase nanoparticles were found to grow from smaller amorphous or anatase clusters. ${ }^{23-30}$ However, a certain amount of brookite phase exists besides the anatase phase during synthesis of nanoparticles. ${ }^{31,32}$ Banfield found the occurrence of brookite on anatase $\{112\}$ twin surfaces in the synthesis of anatase. ${ }^{32}$ Moreover, under higher temperatures and larger 
sizes, the nanoparticles of anatase phase may sinter and transform to rutile phase. ${ }^{31,33,34}$ Cummings et al. $^{35}$ carried out MD simulations of multiple $\mathrm{TiO}_{2}$ nanoparticles with multiple phases and detected phase transformation through simulated X-ray diffraction (XRD) patterns. In previous experimental or MD studies, global parameters, e.g. XRD, X-ray photoelectron spectroscopy (XPS), are frequently utilized to identify phases structure. ${ }^{31-33,35}$ However, detailed local information about nucleation sites and the dynamics of subsequent structure evolutions are still lacking and need to be investigated during the sintering induced phase transformation of small nanoparticles. Fichthorn et al. $^{15}$ developed a local order parameter to identify individual Ti ions as either anatase, rutile, or anatase $\{112\}$ twin-like and tracked the nucleation of a single anatase nanoparticle and nanocrystal aggregates of low-energy Wulff construction via MD simulation. However, the proposed method is unable to identify the brookite phase. Furthermore, in experiments of nanoparticle synthesis, the initial core-shell nanoparticles are more common than the low-energy Wulff construction discussed by Fichthorn et al. ${ }^{15}$

In this study, two typical cases, sintering of two equal-sized core-shell anatase nanoparticles and that of two unequal-sized amorphous and core-shell anatase nanoparticles, are investigated. MD simulation based on semi-empirical force fields is adopted, associated with two characteristic parameterizations, i.e., the coordination number (CN) and the bond angle distribution criteria (BADC), which are defined and utilized to scrutinize the phase and structure evolutions during nanoparticle sintering. Global parameter of simulated XRD patterns is also applied to identify phase 
information at different sintering stages. The dynamic sintering process, the nucleation sites, and the route for the growth of new phase are revealed at an atomistic-scale through our simulation and analysis.

\section{Methods}

\subsection{Simulation Details}

MD simulations are performed using the computer package LAMMPS $^{36}$ to investigate size-dependent structures of $\mathrm{TiO}_{2}$ nanoparticles and phase transformation during sintering. The pair interaction between atoms of $\mathrm{TiO}_{2}$ was proposed by Matsui and Akaogi, ${ }^{37}$ which has been tested as the most suitable force field for reproducing structural properties of $\mathrm{TiO}_{2}$ polymorphs, rutile, anatase, brookite and amorphous phases among the available force fields ${ }^{38,39}$ and widely adopted in MD simulations. ${ }^{5,14,15,19,27,35,40-43}$ The Matsui-Akaogi force field potential can be divided into three parts: repulsion, dispersion, and Coulombic interactions (the first two parts are denoted as the Buckingham potential), which are expressed as Eqn. 1:

$$
U_{i j}=f\left(B_{i}+B_{j}\right) \exp \left(\frac{A_{i}+A_{j}-r_{i j}}{B_{i}+B_{j}}\right)-\frac{C_{i} C_{j}}{r_{i j}{ }^{6}}+\frac{q_{i} q_{j}}{r_{i j}}
$$

where the potential $U_{i j}$ represents the interaction between atom $i$ and atom $j$ with a distance of $r_{i j}$. $q_{i}$ is the effective partial charge of atom $i$ and $q_{\mathrm{O}}$ equals to $-q_{\mathrm{Ti}} / 2$, which are obtained by Taylor et al. ${ }^{44}$ through observing the phonon dispersion of rutile. Other parameters such as $f$ is a standard force of $4.184 \mathrm{~kJ} \cdot \AA^{-1} \cdot \mathrm{mol}^{-1}$. $A_{i}$ and $B_{i}$ are the repulsive radius and softness parameter, which are shown in Table 1. 
Table 1. Interaction Parameters for the Matsui-Akaogi Force Field

\begin{tabular}{ccccc}
\hline & $q(\mathrm{e} \mid)$ & $A(\AA)$ & $B(\AA)$ & \\
& & & & \\
& & & & $\mathrm{C}\left(\AA^{3} \mathrm{~kJ}^{\frac{1}{2}} \mathrm{~mol}^{-1}\right)$ \\
\hline $\mathrm{Ti}$ & +2.196 & 1.1823 & 0.077 & 22.5 \\
$\mathrm{O}$ & -1.098 & 1.6339 & 0.117 & 54.0 \\
\hline
\end{tabular}

Before MD simulations, perfect anatase $\mathrm{TiO}_{2}$ spherical nanoparticles of different sizes $\left(d_{p}=2 \sim 5 \mathrm{~nm}\right)$ are established from the bulk material. The excess titanium atoms or oxygen atoms are removed from the surface to ensure charge balance. ${ }^{40,41}$ Non-periodic boundary conditions are applied in three dimensions and the potential is valid in the whole simulation box without a cutoff truncation in the simulation. The Velocity-Verlet algorithm is employed with a time step of 1 fs to integrate Newton's equations of motion and to update velocities and positions of the atoms. The Nosé-Hoover thermostat is adopted to equilibrate the nanoparticles in the canonical (NVT) ensemble to a target temperature for $3 \times 10^{6}$ timesteps. After that the equilibrated nanoparticles are placed at a center-to-center distance of $4 \mathrm{~nm}$ in the center of the simulation box and the initial velocities of two separated particles are zero. $^{27,35,40,42,43}$ Simulations of the sintering process are in the NVE ensemble, which is based on the fact that both the heat conduction and radiation can be ignored during the sintering process. . $^{5,14,15,27,35,40-43}$

\subsection{Structural Analysis of Nanoparticle}

To distinguish the ordered and disordered structure of nanoparticles, we utilize radical distribution functions (RDF) and $\mathrm{CN}$ to identify global and local structure information of nanoparticles. The definition of RDF, ${ }^{1} g(r)$, is expressed as 


$$
g(r)=\left\langle\frac{d n(r)}{d r} \cdot \frac{1}{4 \pi r^{2} \rho}\right\rangle
$$

where $d n(r)$ is the number of $\mathrm{O}$ atoms in a spherical shell at a distance $r$ from a Ti atom within thickness of $d r . \rho$ is the $\mathrm{O}$ atom number density around a Ti atom in the radius of the Ti-O distance R'. Here structures of equilibrated nanoparticles at different temperatures and sizes are taken as examples for examining the RDF. The high surface-to-volume ratio of nanoparticles leads to decreased melting points as well as a core-shell structure. ${ }^{5}$ Therefore, only Ti atoms in the core region (0-0.5 R, R is the radius of a nanoparticle) are considered, which are indicated by the dashed circle in Figure 1. O atoms within the Ti-O distance of $\mathrm{R}^{\prime}=4 \AA$ are counted, which is enough to include the nearest six $\mathrm{O}$ atoms.

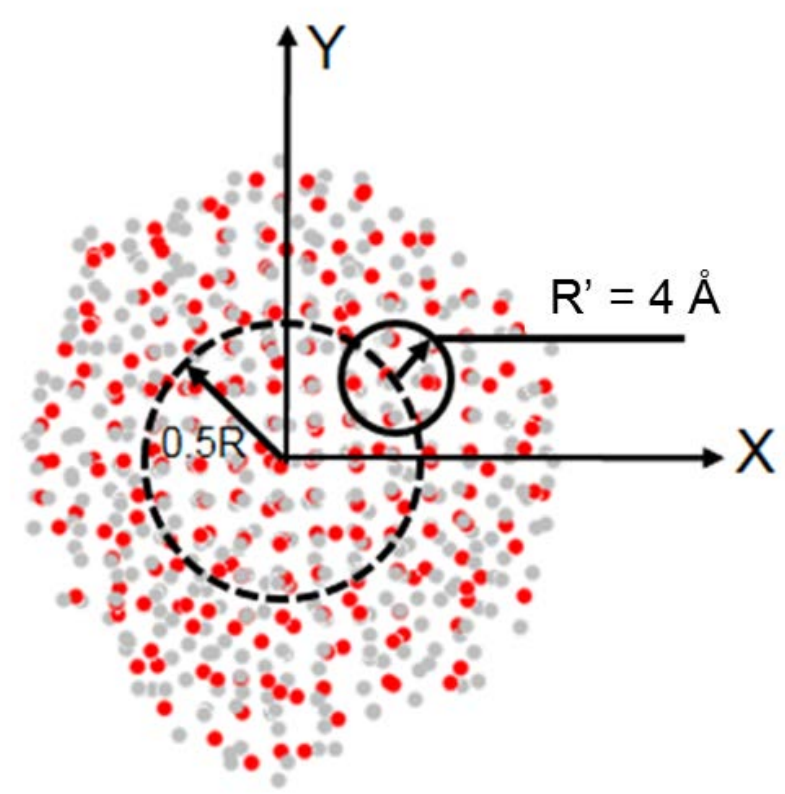

Figure 1. A sketch map indicates the atoms considered in the calculation of the radical distribution function and the coordination number of $\mathrm{Ti}$ atoms in a $\mathrm{TiO}_{2}$ nanoparticle. Red balls are $\mathrm{Ti}$ atoms while grey balls are $\mathrm{O}$ atoms.

The RDFs of nanoparticles at various temperatures and of various sizes are plotted 
in Figure 2 (a) and Figure 2 (b). The first strongest peak in RDF represents the nearest Ti-O bond length, which almost coincides with the ideal bond length of anatase crystal $^{46}$, as shown in the brown dashed line. According to RDFs, $0.24 \mathrm{~nm}$ is chosen to be the criterion for judging whether one $\mathrm{O}$ atom is adjacent to a Ti atom or not. The number of these adjacent $\mathrm{O}$ atoms is then summed up as the $\mathrm{CN}$ of one $\mathrm{Ti}$ atom. Figure 2 (c) and Figure 2 (d) present the mean CNs at various temperatures and of different sizes which correspond to cases in Figure 2 (a) and (b), respectively. The mean CN decreases from 6 to 5.6, which shows a clear global ordered to disordered structure transition with the increase of temperature and the decrease of size. This phenomenon is consistent with the size-dependent structure transition by Zhang et al. ${ }^{5}$
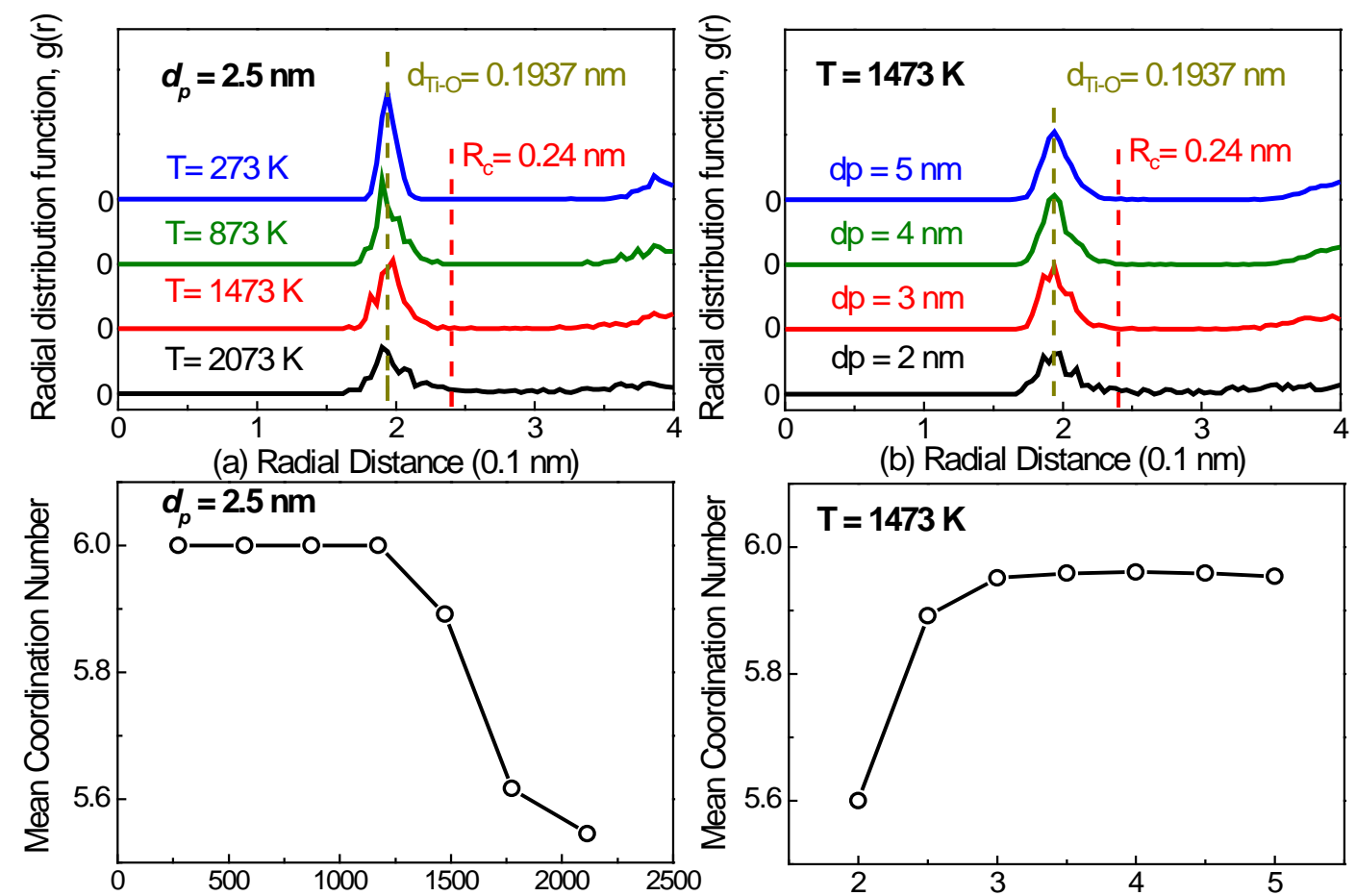

(c) Temperature (K)

(d) Diameter (nm)

Figure 2. The radical distribution functions and coordination number at different temperatures and of varied nanoparticle sizes. (a) RDFs of $d_{p}=2.5 \mathrm{~nm}$ nanoparticles at temperatures ranging from $273 \mathrm{~K}$ to $2073 \mathrm{~K}$. (b) RDFs of nanoparticles of diameters from 2 
$\mathrm{nm}$ to $5 \mathrm{~nm}$ at $T_{0}=1473 \mathrm{~K}$. (c) mean coordination numbers of $d_{p}=2.5 \mathrm{~nm}$ nanoparticles at temperatures ranging from $273 \mathrm{~K}$ to $2073 \mathrm{~K}$. (d) mean coordination numbers of nanoparticles of diameters from $2 \mathrm{~nm}$ to $5 \mathrm{~nm}$ at $\mathrm{T}_{0}=1473 \mathrm{~K}$.

As for local ordered or disordered structure information of one particular atom in a nanoparticle, the $\mathrm{CN}$ of every $\mathrm{Ti}$ atom is calculated to quantify the unique structure. In Figure 3, the $\mathrm{CN}$ of every $\mathrm{Ti}$ atom in a $\mathrm{TiO}_{2}$ nanoparticle is plotted versus the non-dimensional radius position, which shows a core-shell structure of nanoparticles at $d_{p}=3 \sim 5 \mathrm{~nm}$ and an amorphous structure at $d_{p}=2 \mathrm{~nm}$. It should also be noted that the portion of the amorphous layer decreases with the increase of size, but the thicknesses are all within the region of $4 \sim 6 \AA$, which verifies the result of Cummings et al. ${ }^{43}$

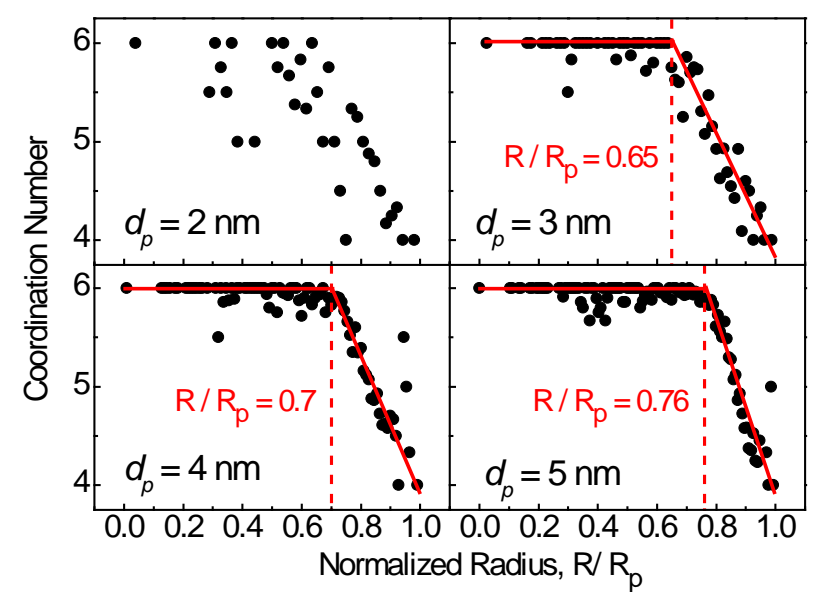

Figure 3. Coordination Number along the nanoparticle radius from the center to the surface of nanoparticles in different sizes, $d_{p}=2,3,4$ and $5 \mathrm{~nm}$ at $1473 \mathrm{~K}$.

\subsection{Structural Characterization of $\mathrm{TiO}_{2}$ Polymorphs}

Simulated XRD ${ }^{1,14,47}$ is used to characterize the global phase structure, which helps 
to distinguish the existing crystal phases and to qualitatively indicate the relative intensity. The simulated XRD patterns can be expressed through the relationship between the diffracted coherent radiation intensity, $I_{k}(b)$, and the diffraction angle, $2 \theta$, determined by applying the Debye functional analysis, ${ }^{48}$

$$
I_{k}(b)=\sum_{n} \sum_{m \neq n} f_{n}(b) f_{m}(b) \frac{\sin \left(2 \pi b r_{n m}\right)}{2 \pi b r_{n m}}
$$

where $f_{n}(b)$ and $f_{m}(b)$ are the scattering factors for atoms $n$ and $m$ separated by a distance of $r_{n m}$. In Eqn. 3, $b=2 \sin (\theta) / \lambda, \lambda$ is the wavelength of the incident X-ray ( $\mathrm{Cu}$ Ka radiation $\lambda=1.5406 \AA) .{ }^{49}$ The simulated XRD patterns for three $\mathrm{TiO}_{2}$ polymorphs are in good agreement with those of experimental results in terms of the diffraction angle and the relative intensity. 


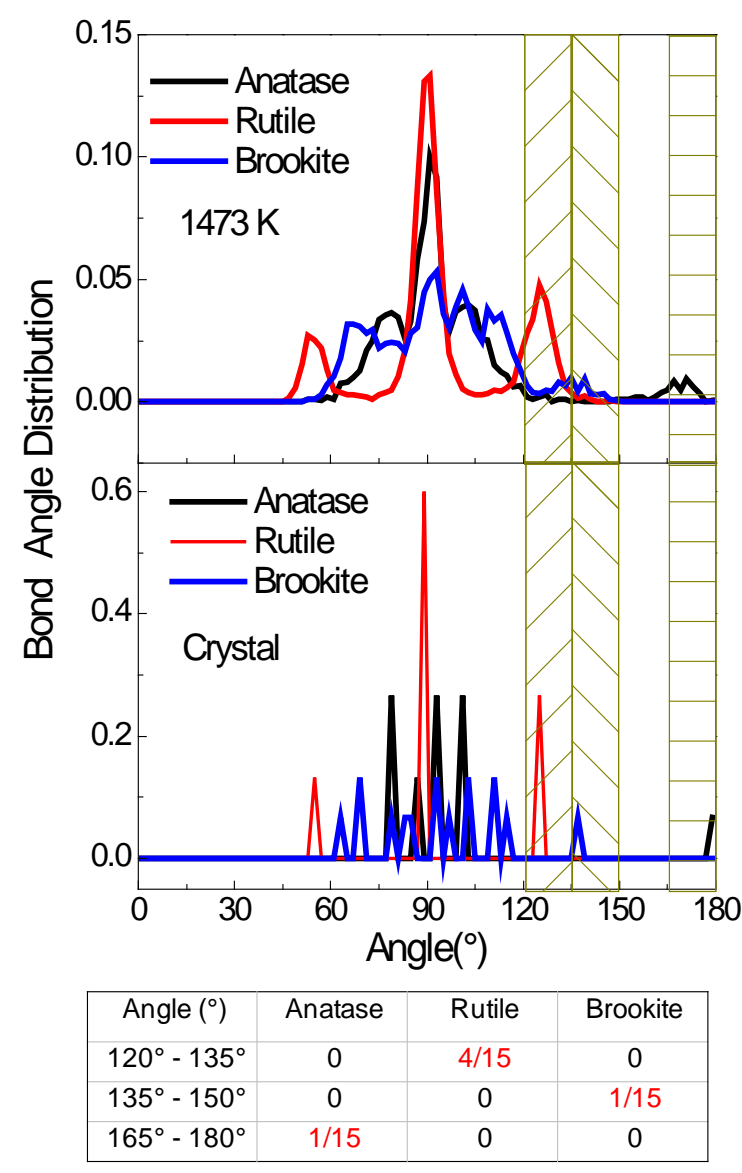

Figure 4. The position and probability of the bond angle formed by a Ti atom and two nearest arbitrary $\mathrm{O}$ atoms of the three polymorphs of $\mathrm{TiO}_{2}$ in equilibrium at $1473 \mathrm{~K}$ and in crystal structure. The values in the table are the criteria integrated from the three regions and used to distinguish different polymorphs.

Moreover, a parameter of the BADC is proposed to distinguish the local Ti-O phase among the three polymorphs of $\mathrm{TiO}_{2}$. In detail, for each $\mathrm{Ti}$ atom surrounded by six nearest $\mathrm{O}$ atoms in the lattice structure of three polymorphs, ${ }^{46}$ the distribution of the O-Ti-O bond angles reflects distinctive local information of the three polymorphs. Figure 4 presents the statistical bond angle distributions for $3 \mathrm{~nm}$ nanoparticles in three phases. It should be noted that the peaks around the regions of $120^{\circ}-135^{\circ}$, 
$135^{\circ}-150^{\circ}, 165^{\circ}-180^{\circ}$ in the bond angle distribution of the ideal crystals and equilibrated nanoparticles are quite different for rutile, brookite, and anatase phases. Therefore, we calculate the ratio of O-Ti-O bond angles in these three regions and define them as the criteria for identifying local phase among three polymorphs. It has been tested that more than $95 \%$ of atoms in the ordered crystal core region can be identified as the corresponding phase for three polymorphs.

\section{Results and Discussions}

Combining global phase information from simulated XRD patterns with local phase information from $\mathrm{CN}$ and BADC, the phase transformation is scrutinized during the sintering processes of two equal-sized anatase nanoparticles and two unequal-sized nanoparticles (amorphous and anatase). Both cases occur frequently during the synthesis of nanoparticles. ${ }^{23-27}$

\subsection{Sintering induced phase transformation of two equal-sized nanoparticles}

According to the previous studies on phase stability of $\mathrm{TiO}_{2}$ nanoparticles, anatase is thermodynamically stable for $d_{p} \leq 2.6 \mathrm{~nm} \cdot{ }^{14,15,22} \mathrm{MD}$ simulation of the sintering process of the two equal-sized anatase nanoparticles is implemented at various nanoparticle sizes and temperatures. Here a typical case is presented with initial temperature of $1473 \mathrm{~K}$ and $d_{p}=2.5 \mathrm{~nm}$, which ensures the core-shell anatase structure of initial nanoparticles. The history of temperature versus time is plotted in Figure 5 on logarithmic coordinates combined with snapshots from cross-section. Atoms are colored according to the regions they locate initially. From Figure 5, the sintering process can be then classified into the following four stages: (i) before the formation 
of the sintering neck, the two nanoparticles first attract with each other and take a lattice rotation to find an appropriate contact site (also regarded as the oriented attachment mechanism in previous studies ${ }^{50-52}$ ), which leads to asymmetric structure evolutions of two nanoparticles as sintering proceeds; (ii) the sintering neck begins to grow from $\mathrm{t}=0.14$ ns to $\mathrm{t}=1$ ns dominated by grain diffusion mechanism which results in the reduction of surface area and the quick increase of temperature; (iii) in the third stage of the sintering from $1 \mathrm{~ns} \sim 10 \mathrm{~ns}$, some atoms in the former grain boundary and surface region diffuse to the initially concave region between nanoparticles, contributing to the neck growth as well as the overall structure from ellipsoid to sphere, accompanied by a temperature rise from about $1600 \mathrm{~K}$ to $1730 \mathrm{~K}$; (iv) finally, the structure of the sintered nanoparticle tends to approach a spherical shape slowly from 10 ns to 140 ns, as the temperature history remains constant before increasing slightly from 40 ns to 120 ns and plateaus after that. The first three stages have been frequently discussed in previous studies, but little work has focused on the last sintering stage due to limitations in computation, which is the focus of this study. 


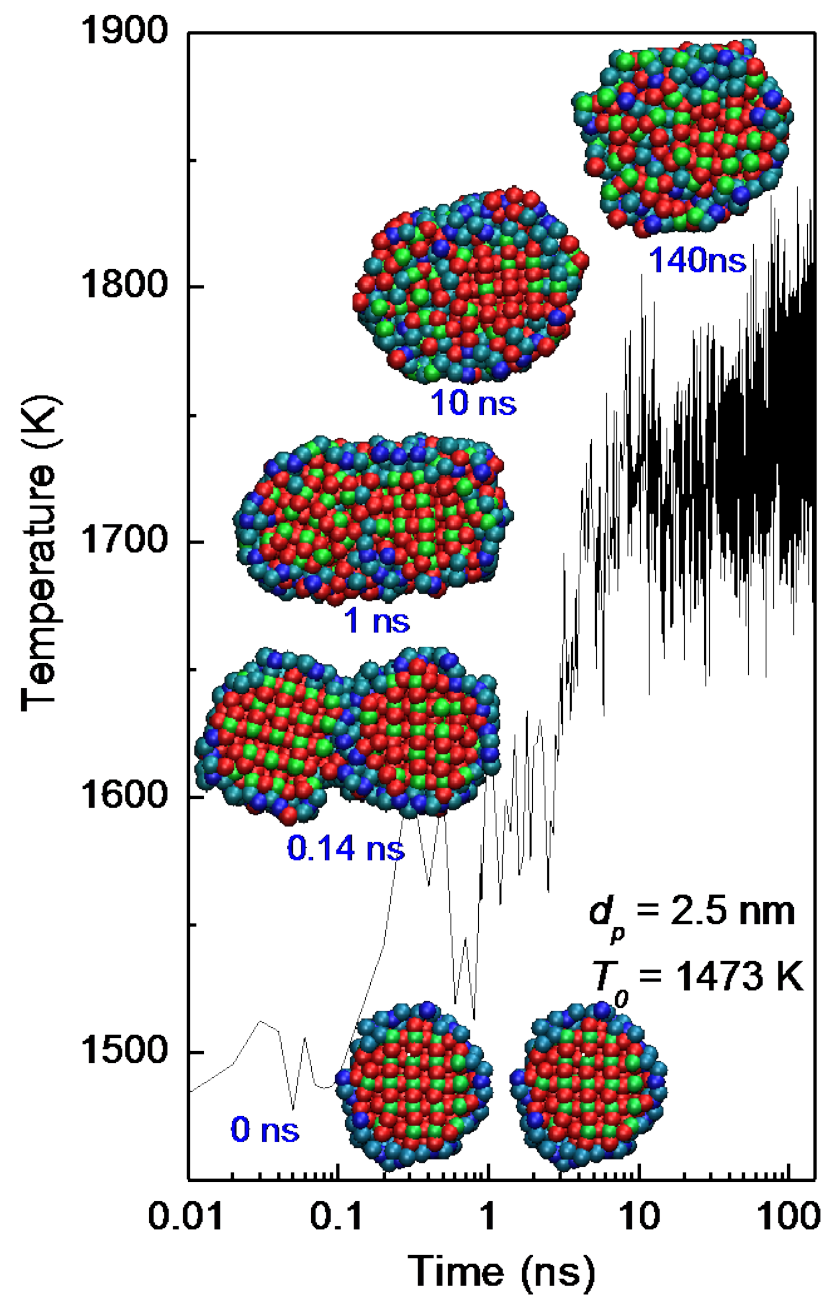

Figure 5. Temporal evolution of temperature and the corresponding morphology changes of two equal-sized anatase from cross-sectional views, with Ti and $O$ atoms initially ( $t=0 \mathrm{~ns}$ ) colored green and red (core) or blue and navy (surface), respectively, during sintering by molecular dynamics simulation in the microconanical ensemble (NVE) at $T_{0}=1473 \mathrm{~K}$.

The simulated XRD patterns are plotted in Figure 6 according to different sintering stages. Compared with that of the standard XRD data of anatase, brookite and rutile from experiments, ${ }^{53}$ the intensities of anatase (101) and anatase (112) decrease, while those of brookite (121) and brookite (241) increase during the last sintering stage. It is noteworthy that the intensity of the brookite (121) increases dramatically from 50 ns to 120 ns and remains much the same after that, which has already been reported in the experiment of the hydrolytic sol-gel synthesis of crystal anatase nanoparticles. ${ }^{54}$ 


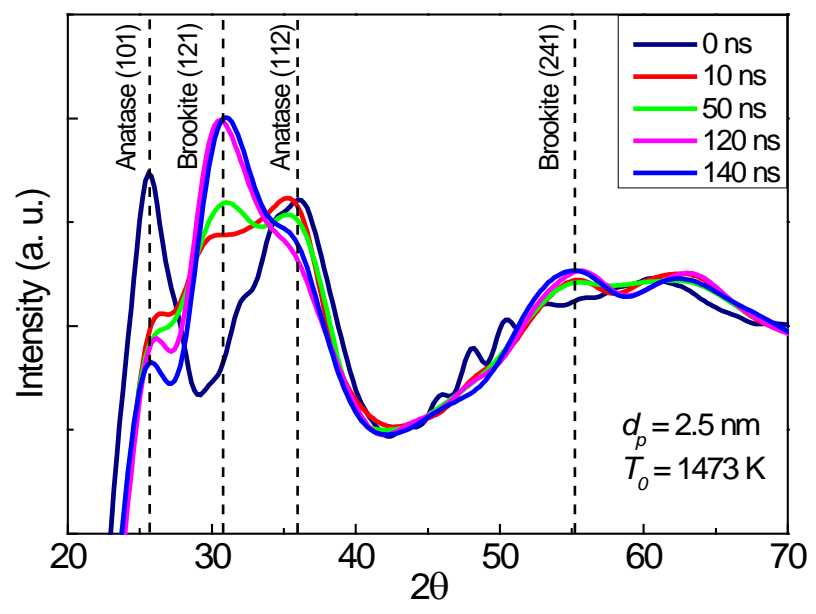

Figure 6. Simulated XRD patterns of the nanoparticles in Figure 5 at different sintering stages at $\mathrm{t}=0$ (navy), 10 (red), 50 (green), 120 (magenta) and $140 \mathrm{~ns}$ (blue) compared with that of the standard data from experiments (dotted lines).

Nevertheless, XRD patterns only present global phase information of nanoparticles. A lack of detailed local information during phase transformation makes it difficult to identify how the nucleation and growth of the new phase take place. Here the CN and the BADC are applied to identify local structure and phase information by observing local Ti-O arrangement. First of all, numbers of Ti atoms in anatase, brookite and rutile phases are counted at the last stage of sintering, as shown in Figure 7, illustrating that atom numbers in anatase and brookite phase enjoy an opposite trend, while few atoms are in rutile phase. Figure 8 presents Ti atoms colored according to local phases in the sintered nanoparticle. In detail, Figure 8 (a) includes all kinds of Ti atoms from the cross-section view, while Figure 8 (b) only presents the Ti atoms in crystal phases. Initially, after the oriented attachment of two sintering nanoparticles, a grain boundary is formed in the contact region. Atoms in the grain boundary are 
amorphous without crystal structures as shown in the blue atoms in Figure 8 (a) and the white gap between two contacting nanoparticles in Figure 8 (b). Before 40 ns, the generation and subsequent disappearance of some small nuclei with a few crystal atoms in brookite phase are observed mainly located on the surface of anatase nanoparticles or in the grain boundary, indicating that the nuclei have not reached the critical nucleus size for the growth of the new phase. Obvious phase transformation begins after $\mathrm{t}=40 \mathrm{~ns}$ and the whole process can be divided into three steps based on three continuous ' $S$ ' curves of the atom numbers in brookite and anatase phases (with slow transformation at the beginning and at the end, but rapid transformation in between) in Figure 7. During the first step, from 40 ns to 50 ns, the size of the brookite nuclei reaches the critical value in the grain boundary region and grows rapidly consuming original atoms in this region. This is consistent with the simulated XRD patterns, with a distinct stronger peak of brookite (121) at t $=50$ ns compared to that at $\mathrm{t}=10 \mathrm{~ns}$ in Figure 6 . The asymmetric structure evolution of two nanoparticles, which results from the oriented attachment, may be amplified because of the heat release during phase transforms from a state with high potential energy to that with low potential energy in the NVE ensemble. These lead to the separated phase transformation steps for the two nanoparticles follow. During the second step of phase transformation from $50 \mathrm{~ns}$ to $70 \mathrm{~ns}$, the atom number in brookite phase fluctuates around a constant value before increasing drastically. The new phase develops into the original core crystal region of the left nanoparticle. Finally, phase transformation engulfs the core region of the right nanoparticle, as the atom number in the brookite 
phase increases sharply once more. It should be noted that the growth of brookite phase in the original core regions of the two nanoparticles develops from nuclei in the grain boundary after the melting of the crystal anatase in two nanoparticles with the rising temperature. Up to now, the majority of atoms in sintered nanoparticles have transformed into brookite phase which can be seen from the changeless brookite (121) peak in simulated XRD patterns and the plateaued atom number of brookite after $\mathrm{t}=$ 120 ns in Figure 6 and Figure 7, respectively. Meanwhile, as the energy of the simulated sintering system is conserved, a slight increase of the temperature from 40 ns to 120 ns leads to the decrease of the potential energy, reflecting the spontaneous characteristic of phase transformation, from a higher energy state to a lower energy state.

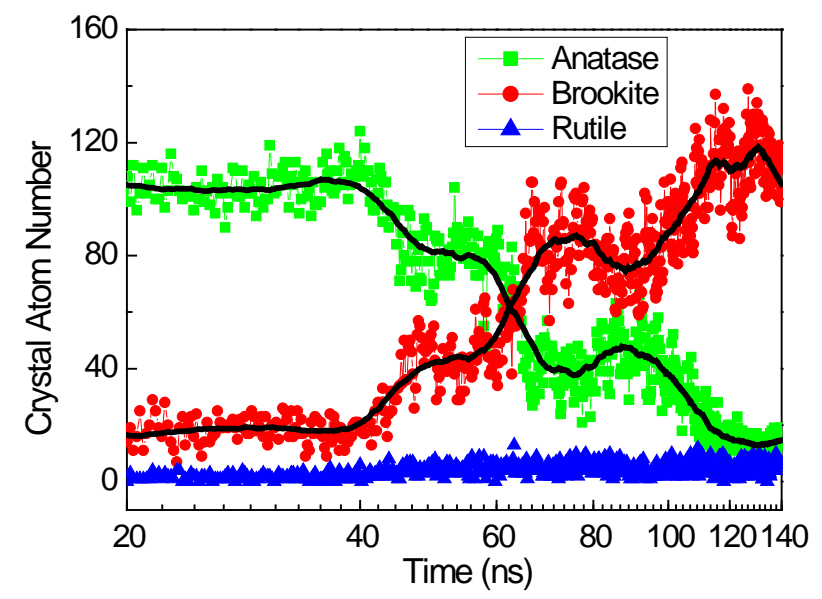

Figure 7. Time evolution of the number of the crystal atoms of the three $\mathrm{TiO}_{2}$ polymorphs (anatase, brookite and rutile) in the last sintering stage from 10 to $140 \mathrm{~ns}$ of the equal-sized sintering process. The dark solid lines indicate the trend of change in the crystal number. 


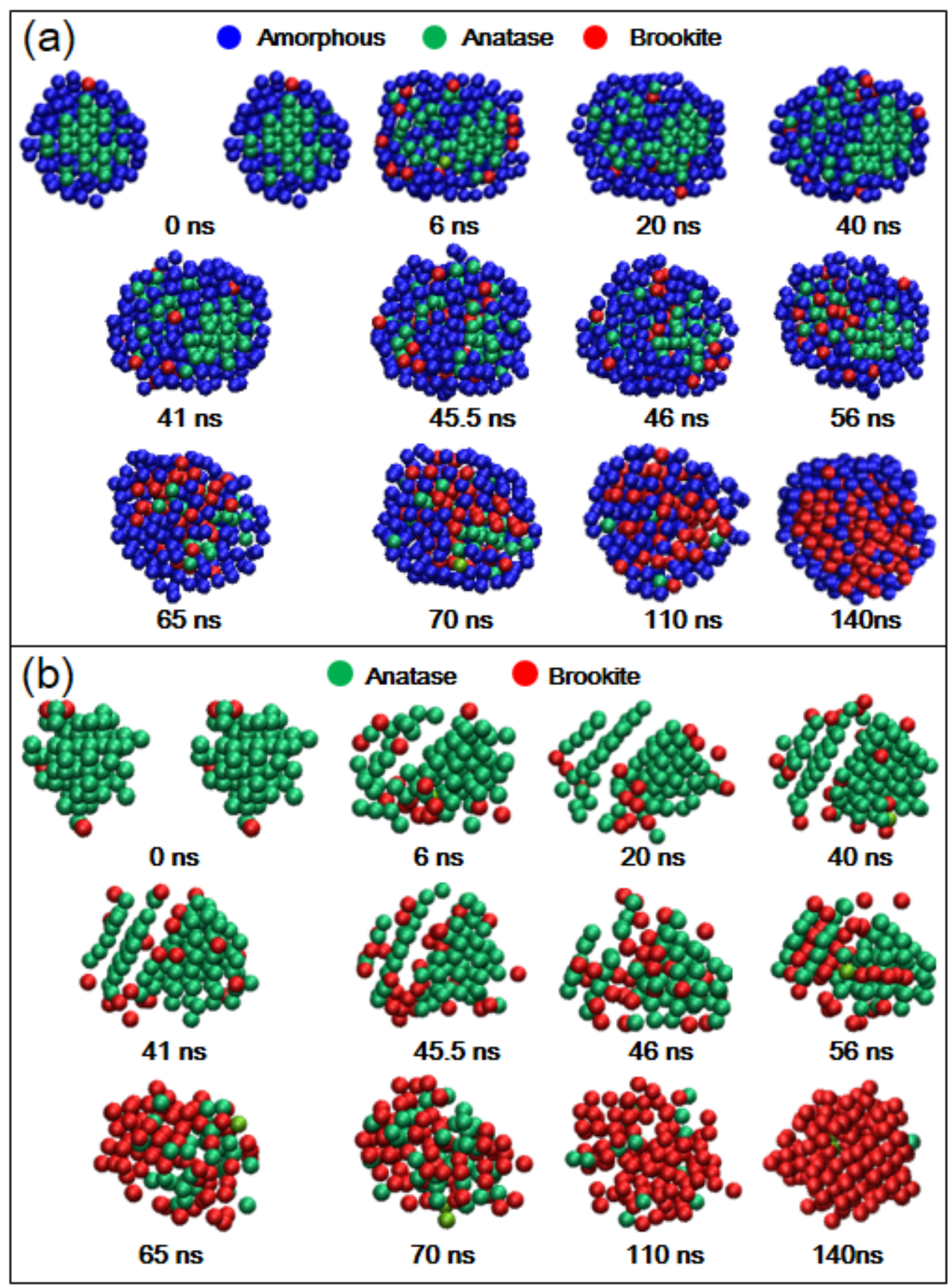

Figure 8. Simulation snapshots of the sintering process of the two equal-sized $2.5 \mathrm{~nm}$ nanoparticles at $T_{0}=1473 \mathrm{~K}$. The local structure information of $T i$ and $O$ atoms are reflected through different colors on $\mathrm{Ti}$ atoms. $\mathrm{Ti}$ atoms in the amorphous structure are colored in blue, and in the crystal structure of anatase are colored in green and brookite in red. (a) The cross-sectional snapshots include all the Ti atoms both in amorphous and crystal structures. (b) The snapshots present all the Ti atoms in the crystal structures. 


\subsection{Sintering induced phase transformation of two unequal-sized nanoparticles}

The sintering case between two unequal-sized nanoparticles is investigated on the same condition. In detail, the unequal-sized case contains the same atom number and sinters at the same initial temperature in the NVE ensemble as the equal-sized case, i.e., $d_{p, 1}=2 \mathrm{~nm}$ with 342 atoms and $d_{p, 2}=2.9 \mathrm{~nm}$ with 1080 atoms at $\mathrm{T}_{0}=1473 \mathrm{~K}$. It should be noted that at this initial temperature, the smaller nanoparticle is amorphous while the larger nanoparticle is core-shell anatase as discussed in Figure 3. The history of temperature versus time is plotted on logarithmic coordinates and the cross-sectional snapshots of morphology evolutions during the sintering process are shown in Figure 9. Ti and $\mathrm{O}$ atoms from two nanoparticles are colored differently. Similar to the equal-sized case, the sintering process can also be divided into four stages: (i) at the first stage, there is a lattice rotation of the larger core-shell nanoparticle before contacting, which is the same as the equal-sized nanoparticle case; (ii) then the contact region between two nanoparticles grows rapidly with a dramatic shape deformation of the smaller nanoparticle and atoms spread out on to the surface of the larger nanoparticle; (iii) from 1 ns to 10 ns, atoms of the smaller nanoparticle continue to spread out, accompanied by the formation of an ellipsoidal nanoparticle as well as a small portion of amorphous atoms nucleated in the contact region; (iv) after $10 \mathrm{~ns}$, some atoms from the smaller nanoparticle mix with the surface atoms of the original larger nanoparticle, while some permeate into the crystal lattice of the larger one, destroying the original ordered structure. 


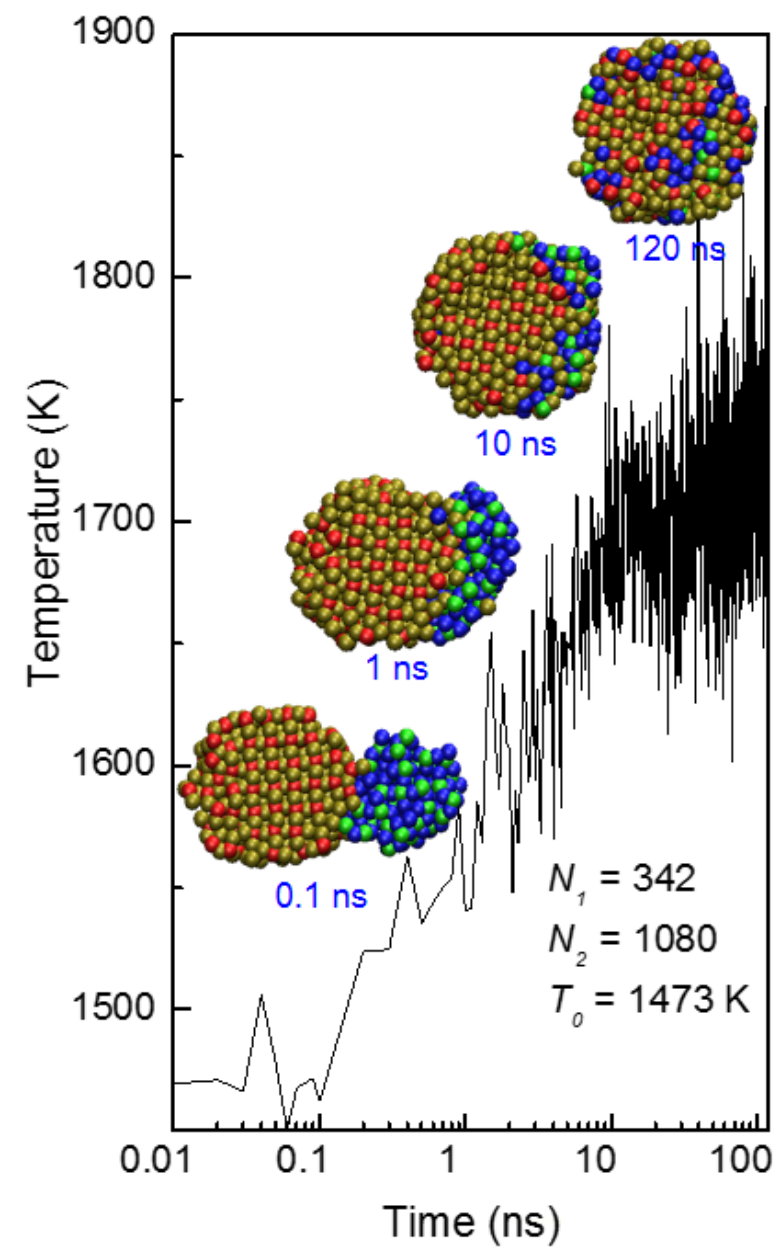

Figure 9. Temporal evolution of the temperature and the corresponding cross-sectional snapshots of the morphologies of two unequal-sized sintering $\mathrm{TiO}_{2}$ nanoparticles $\left(d_{p, 1}=2 \mathrm{~nm}\right.$, Ti: green, $O$ : blue; $d_{p, 2}=2.9 \mathrm{~nm}$, Ti: red, O: gold) by molecular dynamics simulation in the NVE ensemble at $T_{0}=1473 \mathrm{~K}$.

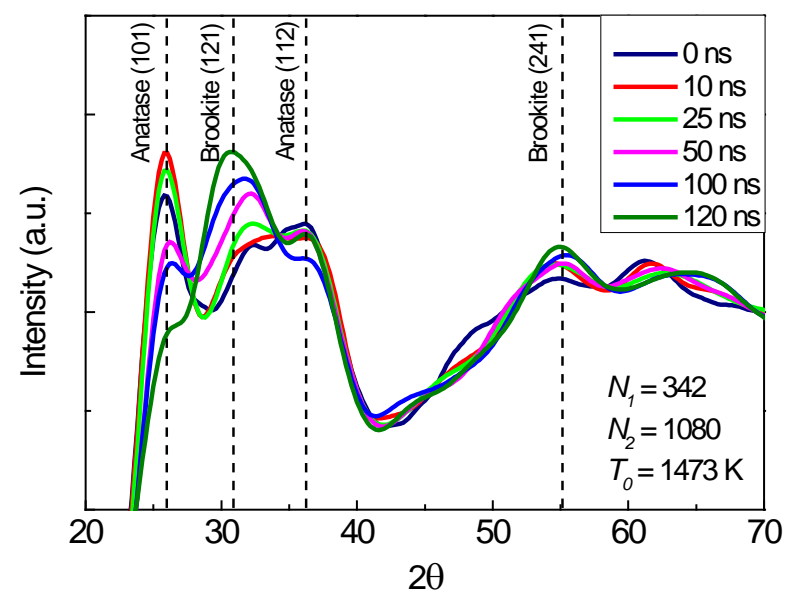

Figure 10. Simulated XRD patterns of the nanoparticles in Figure 9 at different sintering 
stages at $\mathrm{t}=0$ (navy), 10 (red), 25 (green), 50 (magenta), 100 (blue) and 120 ns (olive) compared with that of the standard data from experiments (dotted lines).

To better understand the evolution of atomic arrangements during the sintering of two unequal-sized nanoparticles, the simulated XRD patterns are plotted according to different sintering stages and compared with the standard XRD data of anatase, brookite, and rutile, ${ }^{53}$ as shown in Figure 10. Initially, the intensities of anatase (101) and anatase (211) increase slightly before $10 \mathrm{~ns}$, which indicate that some of the atoms transform to anatase at the third stage of sintering. Then the intensities of anatase (101) and anatase (112) in the simulated XRD patterns decrease significantly, while the phase intensities of brookite (121) and brookite (241) increase at the same time, which indicate that anatase phase transforms into brookite phase.

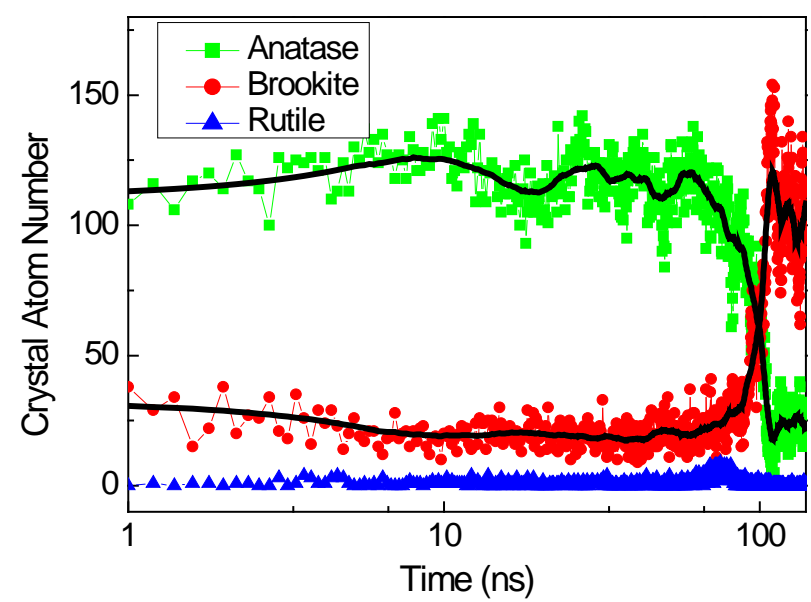

Figure 11. Time evolution of the number of the crystal atoms of $\mathrm{TiO}_{2}$ polymorphs of anatase, brookite and rutile during the sintering from 1 to $140 \mathrm{~ns}$ of the unequal-sized sintering process. The dark solid lines indicate the trend of change in the crystal number.

The CN and the BADC are adopted to identify atoms in amorphous or crystal 
structure as well as different phases and to track the details of phase transformation. The number of $\mathrm{Ti}$ atoms of different crystal phases are counted and plotted in Figure 11. The variation trend of atom number in anatase phase is contrary to that of brookite phase. Phase information of $\mathrm{Ti}$ atoms is expressed in Figure 12 through different colors. Cross-sectional views of the sintering nanoparticles are present in Figure 12 (a) including atoms of amorphous and crystal structures and Figure 12 (b) displays Ti atoms in three crystal polymorphs. The atom number in anatase phase increases slightly before $10 \mathrm{~ns}$. During this period, the original smaller amorphous nanoparticle on the right transforms into crystal anatase phase with an ordered Ti-O arrangement layer by layer. A grain boundary then develops between the newly formed anatase phase and the original crystal anatase, forming a region filled with amorphous atoms. From 10 to 25 ns, a slight rise of temperature leads to a higher diffusion rate of atoms and the melting of the original anatase structure. This consequently destroys the crystal anatase core region and the atom number of anatase phase decreases during this period. Then from 25 ns to 70 ns, the temperature and the atom number in different phases remain much the same. Small nuclei with atoms of brookite phase frequently appear on the surface as well as in the grain boundary before a rapid growth of the new phase. After 70 ns, the temperature trends to rise slightly again, leading to a decrease of the potential energy. From the snapshots in Figure 12, atoms in brookite phase emerge rapidly in the grain boundary region after 85 ns. The brookite phase nuclei in the grain boundary then progress into the newly developed crystal anatase region and the original core region of the larger nanoparticle. No 
obviously separated phase transformation stages have been observed in this case, which is different from the equal-sized sintering case. This is because that the number of atoms in the newly formed anatase phase is fewer compared with that in the original crystal core of the larger nanoparticle and the two stages merge into one step.

Both equal-sized and unequal sized sintering cases indicate that atoms in the grain boundary are important for triggering phase transformation. An explanation of this phenomenon is that the grain boundary region, as a planar zone filled with atoms not in perfect crystal arrangement and relatively weak bonding, provides preferred sites for nucleation and being a breeding ground for the growth of new phases. Besides, compared with the surface nucleation and bulk nucleation, the grain boundary nucleation is temperature independent in kinetics and only determined by the Gibbs free energy of different phases in thermodynamics. ${ }^{28}$ Therefore, phase transformation of the original crystal core regions subsequently develops from the nuclei formed in the grain boundary. 


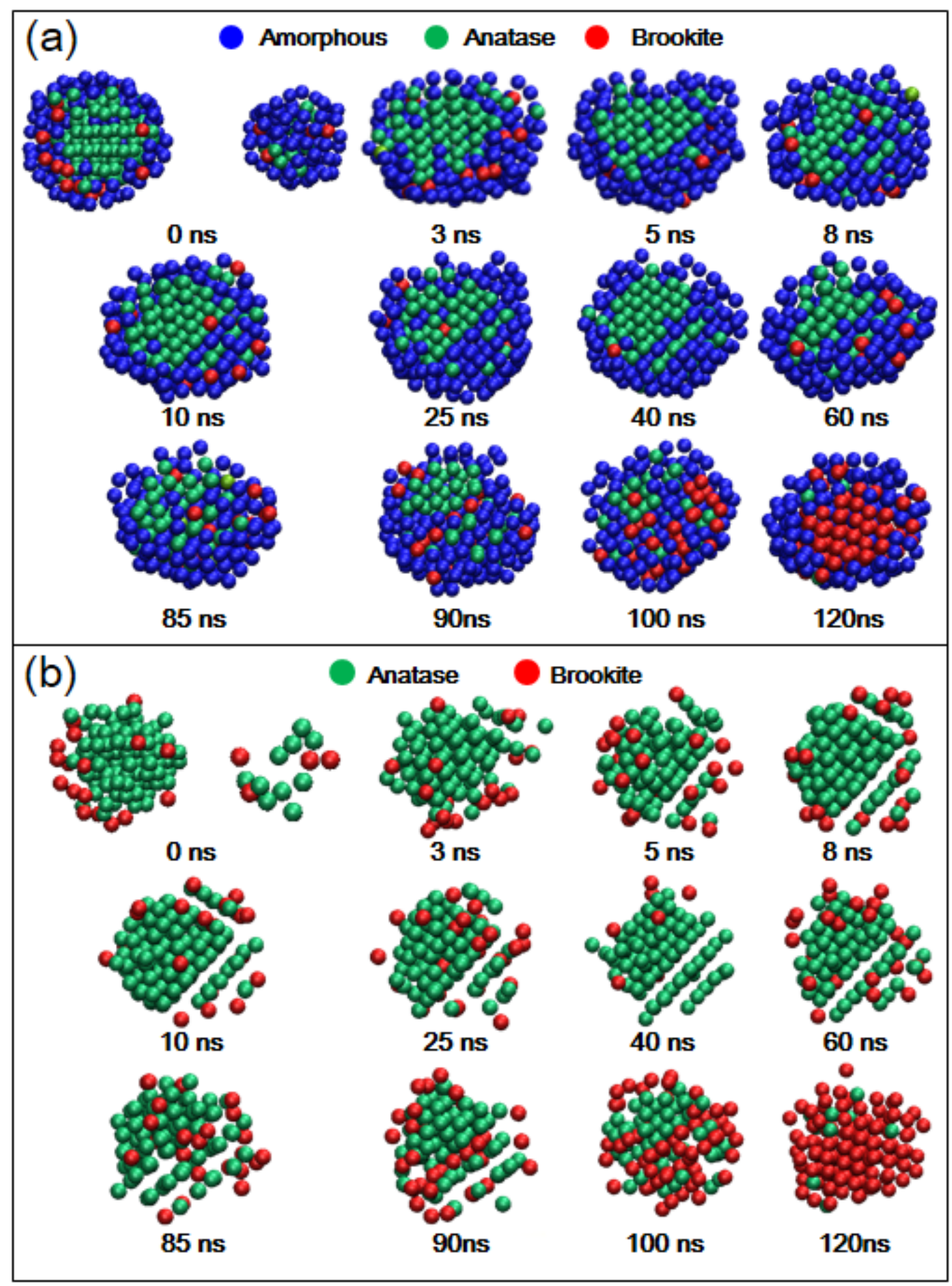

Figure 12. Simulation snapshots of the sintering process of the unequal-sized nanoparticles with $d_{p, 1}=2 \mathrm{~nm}$ and $d_{p, 2}=2.9 \mathrm{~nm}$ at $T_{0}=1473 \mathrm{~K}$. The local structure information of Ti and $O$ atoms are reflected through different colors on Ti atoms. Ti atoms in amorphous and crystal structure of anatase are colored in blue and green, and brookite in red. (a) The cross-sectional snapshots include all the Ti atoms both in amorphous and crystal structures. (b) The snapshots present the Ti atoms in the crystal structures. 
Rutile is more thermodynamically stable than anatase and brookite when $d_{p}$ is larger than $2.6 \mathrm{~nm}^{14,15,22}$, Therefore, as the final sintered nanoparticles of $d_{p} \sim 3.1 \mathrm{~nm}$ in above sintering cases, we believe that they will finally transform to rutile, which is thermodynamically favorable. However, because of the limited simulation time of 140 ns in our simulation, it is unable to capture this successive phase transformation, which may happen later in a much longer time scale. In fact, phase transformation may not take place directly to the most thermodynamically stable state, that is, anatase may firstly transform to brookite then to rutile. According to the Ostwald's step rule, ${ }^{55}$ crystallization often occurs in steps in such a way that often thermodynamically unstable phase occurs first, followed by the thermodynamically stable step. More specifically, if several products are produced in a reaction, it is not the most stable state with the least amount of free energy that is initially obtained, but the least stable one, lying nearest to the original state in free energy. ${ }^{56}$ Stranski and Totomanow ${ }^{57}$ re-examined this rule and argued that the nucleated phase is the phase that has the lowest free-energy barrier of formation, rather than the phase that is globally stable under the conditions prevailing. Our simulations have detected the phase transformation from anatase to brookite, which indicates that the Ostwald's step rule can be applicable to the phase transformation of nano-sized $\mathrm{TiO}_{2}$ particles.

From the kinetic point of view, Penn and Banfield ${ }^{32}$ found that on the sintering of two nanoparticles in initial anatase phase, orientated attachment at anatase surface leads to twinning on $\{112\}$. Brookite, occurs at some $\{112\}$ twin surfaces ${ }^{5}$, may nucleate at twin planes and grow at the expense of anatase. Last but not least, it was 
reported that there were an amount of nanoparticles in brookite and rutile phases during synthesis of ultrafine anatase nanoparticles in experiments, ${ }^{33}$ which support our simulation results to some extent.

\section{Conclusions}

MD simulation, combined with the defined CN and the BADC, elucidates details of the sintering induced phase transformation from the aspect of the local information and reveals the underlying mechanisms. Two typical cases of equal-sized core-shell anatase nanoparticles ( $\left.d_{p}=2.5 \mathrm{~nm}\right)$ and unequal-sized amorphous and core-shell anatase nanoparticles $\left(d_{p, 1}=2 \mathrm{~nm}\right.$ and $\left.d_{p, 2}=2.9 \mathrm{~nm}\right)$, containing the same total atom number, sinter in the NVE ensemble at $\mathrm{T}_{0}=1473 \mathrm{~K}$. The dynamics of phase transformation differ in the above two cases. In the equal-sized case, phase transformation occurs at the final sintering stage of the equal-sized case, which consists of three steps. Atoms in brookite phase first begin to nucleate and grow rapidly at the grain boundary. Original anatase core regions of nanoparticles then melt due to the rising temperature during sintering. The new generated brookite phase subsequently develops into the core regions in stages, forming a larger nanoparticle in ordered atomic arrangement. However, in the unequal-sized case, a distinct phase transformation step has been observed due to different initial structures. Firstly, atoms from the amorphous nanoparticle spread onto the surface of the larger core-shell nanoparticle and nucleate to the crystal anatase phase layer by layer. A grain boundary is then generated between the original core crystal and the newly formed anatase lattice, which serves as the site for the subsequent phase transformation from anatase 
to brookite. Thereafter, phase transformation of the unequal-sized case follows much the same route as the equal-sized case. It is found that grain boundaries formed during sintering trigger the subsequent nucleation and the growth of new phase and the initial structures of two nanoparticles, i.e. core-shell or amorphous structure, significantly influence the dynamics of sintering induced phase transformation. However, for the final stable phase of the sintered nanoparticles in our simulations, we believe that they will transform to rutile, which is more thermodynamically stable than anatase and brookite when nanoparticles are larger than $2.6 \mathrm{~nm}$. Moreover, our simulation results confirm that the Ostwald's step rule is applicable to the phase transformation of nano-sized $\mathrm{TiO}_{2}$ particles. That is, anatase may firstly transform to brookite then to rutile, rather than directly to the most stable state.

\section{Acknowledgements}

Support from the Major Project of the National Science Foundation of China (Grant No. 51390493) and the Center for Combustion Energy at Tsinghua University is gratefully acknowledged. The simulations were partly performed on the Tsinghua High-Performance Parallel Computer supported by the Tsinghua National Laboratory for Information Science and Technology and partly on ARCHER funded under the EPSRC project "UK Consortium on Mesoscale Engineering Sciences (UKCOMES)” (Grant No. EP/L00030X/1).

\section{References}


(1) Zhang, H.; Banfield, J. F. Structural Characteristics and Mechanical and Thermodynamic Properties of Nanocrystalline $\mathrm{TiO}_{2}$. Chem. Rev. 2014, 114, 9613-9644.

(2) Samanta, A.; Tuckerman, M. E.; Yu, T. Q.; Weinan, E. Microscopic Mechanisms of Equilibrium Melting of a Solid. Science 2014, 346, 729-732.

(3) Binder, K. Theory of First-Order Phase Transitions. Rep. Prog. Phys. 1987, 50, 783.

(4) Navrotsky, A. Nanoscale Effects on Thermodynamics and Phase Equilibria in Oxide Systems. J. Chem. Thermodyn. 2007, 39, 1-9.

(5) Zhang, Y. Y.; Li, S. Q.; Yan, W.; Tse, S. D. Effect of Size-Dependent Grain Structures on the Dynamics of Nanoparticle Coalescence. J. Appl. Phys. 2012, 111, 124321.

(6) Kofman, R.; Cheyssac, P.; Aouaj, A.; Lereah, Y.; Deutscher, G.; Ben-David, T.; Penisson, J. M.; Bourret, A. Surface Melting Enhanced by Curvature Effects. Surf. Sci. 1994, 303, 231-246.

(7) Buffat, P.; Borel, J. P. Size Effect on the Melting Temperature of Gold Particles. Phys. Rev. A 1976, $13,2287$.

(8) Baletto, F.; Ferrando, R. Structural Properties of Nanoclusters: Energetic, Thermodynamic, and Kinetic Effects. Rev. Mod. Phys. 2005, 77, 371.

(9) McHale, J. M.; Auroux, A.; Perrotta, A. J.; Navrotsky, A. Surface Energies and Thermodynamic Phase Stability in Nanocrystalline Aluminas. Science 1997, 277, 788-791.

(10) Su, W.; Zhang, J.; Feng, Z.; Chen, T.; Ying, P.; Li, C. Surface Phases of $\mathrm{TiO}_{2}$ Nanoparticles Studied by UV Raman Spectroscopy and FT-IR Spectroscopy. J. Phys. Chem. C 2008, 112, 7710-7716.

(11) Zhang, H.; Banfield, J. F. Understanding Polymorphic Phase Transformation Behavior during Growth of Nanocrystalline Aggregates: Insights from $\mathrm{TiO}_{2}$. J. Phys. Chem. B 2000, 104, 3481-3487.

(12) Cheng, H.; Ma, J.; Zhao, Z.; Qi, L. Hydrothermal Preparation of Uniform Nanosize Rutile and Anatase Particles. Chem. Mater. 1995, 7, 663-671.

(13) Yang, J.; Mei, S.; Ferreira, J. M. F. Hydrothermal Synthesis of Nanosized Titania Powders: Influence of Peptization and Peptizing Agents on the Crystalline Phases and Phase Transitions. J. Am. Ceram. Soc. 2000, 83, 1361-1368.

(14) Naicker, P. K.; Cummings, P. T.; Zhang, H.; Banfield, J. F. Characterization of Titanium Dioxide Nanoparticles using Molecular Dynamics Simulations. J. Phys. Chem. B 2005, 109, 15243-15249.

(15) Zhou, Y.; Fichthorn, K. A. Microscopic View of Nucleation in the Anatase-to-Rutile Transformation. J. Phys. Chem. C 2012, 116, 8314-8321.

(16) Hummer, D. R.; Kubicki, J. D.; Kent, P. R.; Post, J. E.; Heaney, P. J. Origin of Nanoscale Phase Stability Reversals in Titanium Oxide Polymorphs. J. Phys. Chem. C 2009, 113, 4240-4245.

(17) Barnard, A. S.; Zapol, P. Effects of Particle Morphology and Surface Hydrogenation on the Phase Stability of TiÖ. Phys. Rev. B 2004, 70, 235403.

(18) Finnegan, M. P.; Zhang, H.; Banfield, J. F. Phase Stability and Transformation in Titania Nanoparticles in Aqueous Solutions Dominated by Surface Energy. J. Phys. Chem. C 2007, 111, 1962-1968.

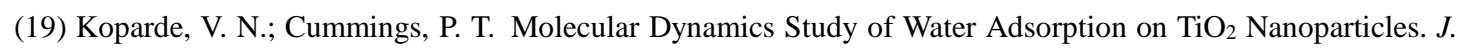
Phys. Chem. C 2007, 111, 6920-6926.

(20) Ricci, P. C.; Carbonaro, C. M.; Stagi, L.; Salis, M.; Casu, A.; Enzo, S.; Delogu, F. Anatase-to-Rutile Phase Transition in $\mathrm{TiO}_{2}$ Nanoparticles Irradiated by Visible Light. J. Phys. Chem. C 2013, 117, 7850-7857.

(21) Barnard, A. S.; Zapol, P. A Model for the Phase Stability of Arbitrary Nanoparticles as a Function of Size and Shape. J. Chem. Phys. 2004, 121, 4276-4283.

(22) Hummer, D. R.; Kubicki, J. D.; Kent, P. R. C.; Post, J. E.; Heaney, P. J. Origin of Nanoscale Phase Stability Reversals in Titanium Oxide Polymorphs. J. Phys. Chem. C 2009, 113, 4240-4245.

(23) Xing, Y.; Köylü, Ü. Ö.; Rosner, D. E. Synthesis and Restructuring of Inorganic Nano-Particles in Counterflow 
Diffusion Flames. Combust. Flame. 1996, 107, 85-102.

(24) Li, G. S.; Li, L. P.; Boerio-Goates, J.; Woodfield, B. F. High Purity Anatase $\mathrm{TiO}_{2}$ Nanocrystals: Near Room-Temperature Synthesis, Grain Growth Kinetics, and Surface Hydration Chemistry. J. Am. Chem. Soc. 2005, 127, 8659-8666.

(25) Zhao, B.; Uchikawa, K.; McCormick, J. R.; Ni, C. Y.; Chen, J. G.; Wang, H. Ultrafine Anatase $\mathrm{TiO}_{2}$ Nanoparticles Produced in Premixed Ethylene Stagnation Flame at 1atm. Proc. Combust. Inst. 2005, 30, 2569-2576.

(26) Arabi-Katbi, O. I.; Pratsinis, S. E.; Morrison, P. W.; Megaridis, C. M. Monitoring the Flame Synthesis of $\mathrm{TiO}_{2}$ Particles by in-Situ FTIR Spectroscopy and Thermophoretic Sampling. Combust. Flame. 2001, 124, 560-572.

(27) Wang, J.; Li, S.; Yan, W.; Tse, S. D.; Yao, Q. Synthesis of $\mathrm{TiO}_{2}$ Nanoparticles by Premixed Stagnation Swirl Flames. Proc. Combust. Inst. 2011, 33, 1925-1932.

(28) Zhang, H.; Banfield, J. F. Kinetics of Crystallization and Crystal Growth of Nanocrystalline Anatase in Nanometer-Sized Amorphous Titania. Chem. Mat. 2002, 14, 4145-4154.

(29) Ivashchenko, V. I.; Turchi, P. E. A.; Shevchenko, V. I. Simulations of Indentation-Induced Phase Transformations in Crystalline and Amorphous Silicon. Phys. Rev. B 2008, 78, 035205.

(30) Levitas, V. I.; Ren, Z.; Zeng, Y.; Zhang, Z.; Han, G. Crystal-Crystal Phase Transformation via Surface-Induced Virtual Premelting. Phys. Rev. B 2012, 85, 220104.

(31) Ding, X. Z.; Liu, X. H. Grain Growth Enhanced by Anatase-to-Rutile Transformation in Gel-Derived Nanocrystalline Titania Powders. J. Alloys Compd. 1997, 248, 143-145.

(32) Penn, R. L., \& Banfield, J. F. Oriented Attachment and Growth, Twinning, Polytypism, and Formation of Metastable Phases: Insights from Nanocrystalline $\mathrm{TiO}_{2}$. Am. Mineral. 1998, 83(9-10), 1077-1082.

(33) McCormick, J. R.; Zhao, B.; Rykov, S. A.; Wang, H.; Chen, J. G. Thermal Stability of Flame-Synthesized Anatase $\mathrm{TiO}_{2}$ Nanoparticles. J. Phys. Chem. B 2004, 108, 17398-17402.

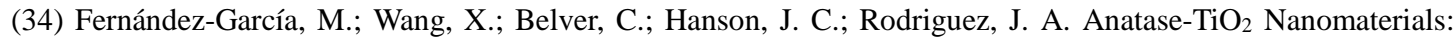
Morphological/Size Dependence of the Crystallization and Phase Behavior Phenomena. J. Phys. Chem. C 2007, 111, 674-682.

(35) Koparde, V. N.; Cummings, P. T. Phase Transformations during Sintering of Titania Nanoparticles. ACS nano 2008, 2, 1620-1624.

(36) Plimpton, S. Fast Parallel Algorithms for Short-Range Molecular Dynamics. J. Comp. Phys. 1995, 117, 1-19.

(37) Matsui, M.; Akaogi, M. Molecular Dynamics Simulation of the Structural and Physical Properties of the Four Polymorphs of $\mathrm{TiO}_{2}$. Mole. Simul. 1991, 6, 239-244.

(38) Swamy, V.; Gale, J. D. Transferable Variable-Charge Interatomic Potential for Atomistic Simulation of Titanium Oxides. Phys. Rev. B 2000, 62, 5406.

(39) Swamy, V.; Gale, J. D.; Dubrovinsky, L. S. Atomistic Simulation of the Crystal Structures and Bulk Moduli of $\mathrm{TiO}_{2}$ Polymorphs. J. Phys. Chem. Solids 2001, 62, 887-895.

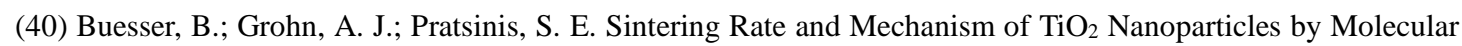
Dynamics. J. Phys. Chem. C 2011, 115,11030-11035.

(41) Zhang, Y. Y.; Li, S. Q.; Yan, W.; Yao, Q.; Tse S. D. Role of Dipole-Dipole Interaction on Enhancing Brownian Coagulation of Charge-Neutral Nanoparticles in the Free Molecular Regime. J. Chem. Phys. 2011, 134, 084501.

(42) Koparde, V. N.; Cummings, P. T. Sintering of Titanium Dioxide Nanoparticles: a Comparison between Molecular Dynamics and Phenomenological Modeling. J. Nanopart. Res. 2008, 10, 1169-1182.

(43) Koparde, V. N.; Cummings, P. T. Molecular Dynamics Simulation of Titanium Dioxide Nanoparticle Sintering. J. Chem. Phys. B, 2005, 109, 24280-24287.

(44) Traylor, J. G.; Smith, H. G.; Nicklow, R. M.; Wilkinson, M. K. Lattice Dynamics of Rutile. Phys. Rev. B 1971, 
3, 3457

(45) Hendy, S.; Brown, S. A.; Hyslop, M. Coalescence of Nanoscale Metal Clusters: Molecular-Dynamics Study. Phys. Rev. B 2003, 68, 241403.

(46) Mo, S. D.; Ching, W. Y. Electronic and Optical Properties of Three Phases of Titanium Dioxide: Rutile, Anatase, and Brookite. Phys. Rev. B 1995, 51, 13023.

(47) Barnard, A. S.; Zapol, P. A Model for the Phase Stability of Arbitrary Nanoparticles as a Function of Size and Shape. J. Chem. Phys. 2004, 121, 4276-4283.

(48) Kazakov, A. V.; Shpiro, E. S.; Voskoboinikov, T. V. Application of Debye Function Analysis to Particle Size and Shape Determination in Ir/SiO 2 Catalysts. J. Phys. Chem. 1995, 99, 8323-8327.

(49) Cromer, D. T.; Mann, J. B. X-ray Scattering Factors Computed from Numerical Hartree-Fock Wave Functions. Acta Crystallogr., Sect. A: Cryst. Phys., Diffr., Theor. Gem Crystallogr. 1968, 24, 321-324.

(50) Zhu, H. Sintering Processes of Two Nanoparticles: a Study by Molecular Dynamics Simulations. Philos. Mag. Lett. 1996, 73, 27-33.

(51) Arcidiacono, S.; Bieri, N. R.; Poulikakos, D.; Grigoropoulos, C. P. On the Coalescence of Gold Nanoparticles. Int. J. Multiphase Flow 2004, 30, 979-994.

(52) Iijima, S.; Ajayan, P. M. Substrate and Size Effects on the Coalescence of Small Particles. J. Appl. Phys. 1991, 70, 5138-5140.

(53) Wang, C.; Deng, Z. X.; Li, Y. The Synthesis of Nanocrystalline Anatase and Rutile Titania in Mixed Organic Media. Inorg. Chem. 2001, 40, 5210-5214.

(54) Zhang, H.; Finnegan, M.; Banfield, J. F. Preparing Single-Phase Nanocrystalline Anatase from Amorphous Titania with Particle Sizes Tailored by Temperature. Nano Lett. 2001, 1, 81-85.

(55) Ostwald, W. Z. Studies on Formation and Transformation of Solid Materials. Z. Phys. Chem. 1897, 22, 289-330.

(56) Van Santen, R. A. The Ostwald Step Rule. J. Phys. Chem. 1984, 88, 5768-5769.

(57) Stranski, I. N.; Totomanow, D. Rate of Formation of (crystal) Nuclei and the Ostwald Step Rule. Z. Phys. Chem. 1933, 163, 399-408.

(58) Banfield, J. F.; Veblen, D. R. Conversion of Perovskite to Anatase and $\mathrm{TiO}_{2}$ (B): A TEM Study and the Use of Fundamental Building Blocks for Understanding Relationships among the $\mathrm{TiO}_{2}$ Minerals. Am. Mineral. 1992, 77, 545-557.

(59) Gribb, A. A.; Banfield, J. F. Particle Size Efects on Transformation Kinetics and Phase Stability in Nanocrystalline $\mathrm{TiO}_{2}$. Am. Mineral. 1997, 82, 717-728.

\section{Table of Contents}

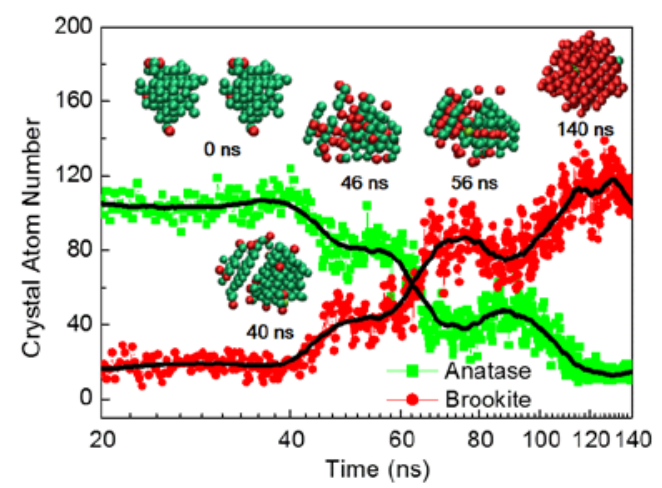

Time evolution of the number of the crystal atoms of $\mathrm{TiO}_{2}$ polymorphs in the last sintering 
stage of two equal-sized core-shell anatase nanoparticles at $T_{0}=1473 \mathrm{~K}$, combined with simulation snapshots presenting $\mathrm{Ti}$ atoms in anatase and brookite phases. 